(C) Elsevier Sequoia S.A., Lausanne - Printed in The Netherlands

\title{
A RHEOLOGICAL MECHANISM OF PENETRATIVE WEAR
}

\author{
T. R. BATES, Jr, and K. C. LUDEMA
}

Department of Mechanical Engineering, University of Michigan, Ann Arbor, Michigan 48104 (U.S.A.) W. A. BRAINARD

National Aeronautic and Space Administration, Lewis Research Center, Cleveland, Ohio 44135 (U.S.A.) (Received July 17, 1974)

\section{SUMMARY}

A model is proposed which explains the penetrative wear of a soft material by a harder one. Three distinct modes of penetration are present depending on the applied load. During the most severe penetration plate-like wear debris is ejected at the leading edge of the slider. A scrics of slip linc fields is presented to approximate this debris formation process. Plastic constraint is seen to be an important factor in wear particle formation.

\section{INTRODUCTION}

While the problem of dry friction and wear is of great technological interest, there has been surprisingly little research done to develop models for the mechanics of the process. Although Tabor ${ }^{1}$ related frictional properties to the shear strengths of the interface and the substrates, no definitive work has been published relating the wear process to mechanical strengths. Archard ${ }^{2}$ has proposed a widely quoted wear equation:

$$
V=\frac{K L}{H} D
$$

where $V$ is the volume of material removed; $L$ is the load; $D$ is the distance slid; $H$ is the hardness of the softer material and $K$ is a "constant" peculiar to the operating conditions of the system. The wear mechanism associated with this equation is ill defined, however, and the values of $K$ are so widely scattered that the theory becomes questionable in value. Antler ${ }^{3}$ and Cocks $^{4.5}$ each made critical observations concerning prow and wedge formation that were never resolved into a viable theory. In addition, $\operatorname{Suh}^{6.7}$ and his co-workers have offered a delamination theory which attempts to relate wear particle shape to dislocation behavior in the substrate. While the above theories have contribuled important concepts none has related wear mechanics to material strengths in convincing fashion.

Recently, however, Bill and Wisander ${ }^{8}$ published a model for the wear of copper on bearing steel. In it they outlined a slip line field for the migration of 
copper to the exit surface of the wear scar and the formation of plate-like debris at the trailing edge of the contact zone. The research in the present paper is a refinement of the slip line model based on scaled-up experiments done with modeling clay and wax as well as with pin-on-disk experiments conducted in a scanning electron microscope.

One factor which complicates the wear literature is the use of such ambiguous terms as abrasive and adhesive wear. While adhesive wear generally connotes metal to metal contact and abrasive wear implies use of a very hard grit neither term is defined in terms of the mechanics of wear. For example, Antler ${ }^{3}$ has shown that wear of copper, gold and aluminum pins on similar flats can generate a severely work hardened prow on the pin. This prow proceeds to gouge out the surface of the flat. This type of wear would conventionally be called severe adhesive wear and yet the gouging process is very similar to what is generally called abrasion. For this reason a new mechanistically defined term is proposed-penetrative wear. The term penetrative wear is meant to classify all those situations where a locally harder surface penetrates and wears a locally softer surface. This would include classic abrasion of a soft material by abrasive paper but only in those cases where the load on the individual grit is sufficient to cause penetration. This definition would also include wear resulting from local variations in hardness due to work hardening, impurities, or phase difference which cause penetration on contact. The mechanics of penetrative wear debris formation are explained later in terms of the plastic constraint imposed by the penetrating surface and the flow properties of the softer material.

\section{EXPERIMENTAL METHODS}

In studies of wear mechanisms it is most desirable to observe the processes while they occur rather than afterward, if possible. Because of the small scale of the events this has not been possible in the past. However, a pin-on-disk machine mounted in a scanning electron microscope (described clscwhere ${ }^{9}$ ) allows the wear particle formation process to be photographed and video taped as it occurs. In order to describe the subsurface events that occur it is necessary to enlarge the scale with more traditional modeling techniques.

\section{In situ SEM experiments}

The pin for this work was a commercial grade 25 micrometer diameter hemispherically tipped diamond cone with a 75 degree included angle. Since the penetration in all cases was not deep enough to cause contact with the conical surface the slider can be considered hemispherical. The diamond was mounted in a steel holder and inserted in a flexible beam loading device ${ }^{9}$, (see Fig. 1). This unit gave both normal and transverse load measuring capability. Both steel and cast iron disks were used in this set of experiments. The steel disks were machined from $15 \mathrm{~mm}$ diam., $4 \mathrm{~mm}$ thick AISI 1075 round stock which had been austenitized at $760^{\circ} \mathrm{C}$ for one hour in a salt bath, oil quenched, and tempered at $370^{\circ} \mathrm{C}$ for one hour. The white cast iron disks were electrical discharge machined from $50 \mathrm{~mm}$ thick sections, as cast. The composition of the white iron as supplied by Climax Molybdenum Company was approximately $2.9 \mathrm{C}, 0.75 \mathrm{Mn}$, 


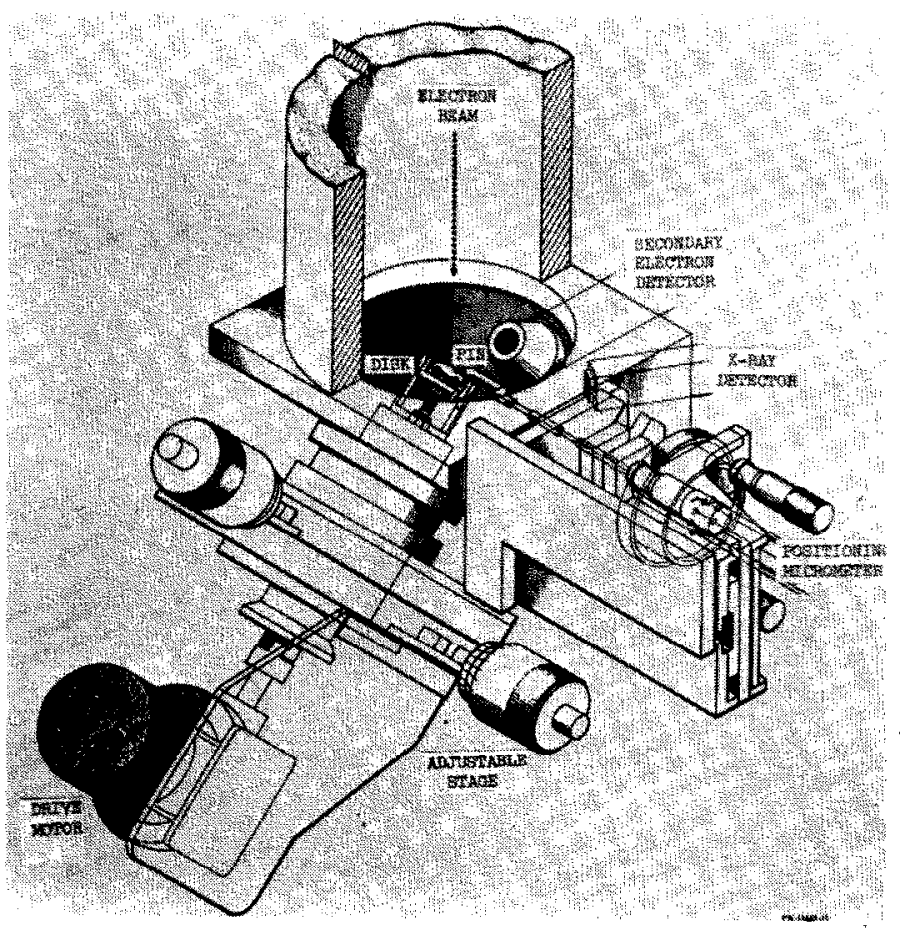

Fig. 1. Pin-on disk wear machine mounted in a scanining electron microscope.

$0.65 \mathrm{Si}$, $17.5 \mathrm{Cr}$, with the balance being iron. The pearlitic matrix, carbide distribution, hardness, and heat treatments are described in another paper ${ }^{10}$. The heat treatment for the 1075 steel was selected to give approximately the same hardness and microstructure as the matrix of the white iron. Both types of disks were run in the scanning electron microscope chamber with varying loads on the diamond stylus to determine different wear regimes.

\section{Modeling experiments}

In order to approximate the flow underneath the diamond indentor it was decided to enlarge the scale of the experiments and slide a $50 \mathrm{~mm}$ diam., $25 \mathrm{~mm}$ thick brass cylinder over the surface of gridded modeling clay and paraffin wax. The molded clay and cast wax were constrained by the lubricated sides of the modeling fixture shown in Fig. 2. This constraint along with the matching thickness of the brass cylinder, simulated the plane strain conditions that exist in the disk at the center-line of contact. One of the sides of the modeling fixture was transparent allowing both still photography and video tape to capture the sequence of events in the formation of a wear particle.

\section{RESULTS}

Observation of the behavior of diamond on steel and diamond in the matrix of white iron leads to the definition of three distinct wear regimes relative 


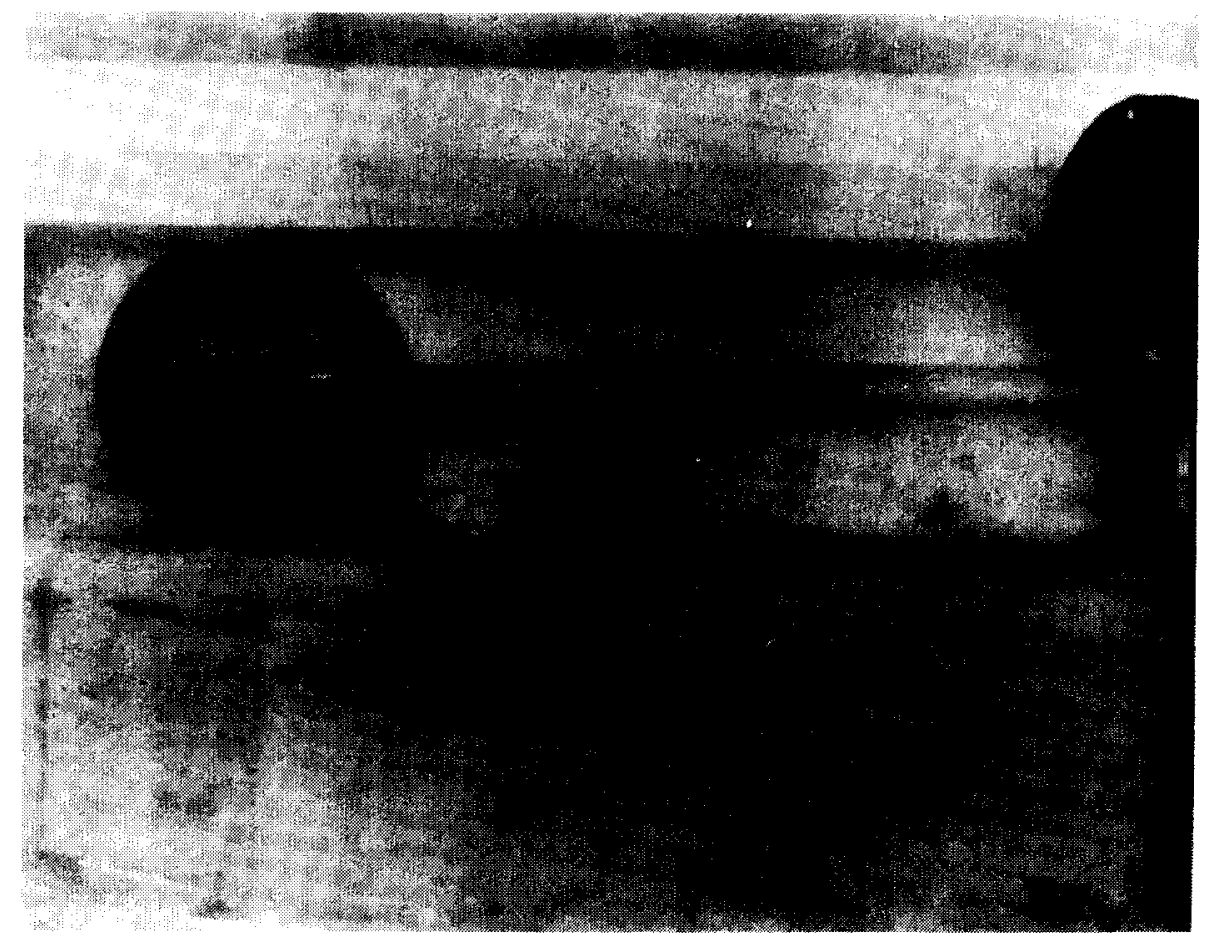

Fig. 2. Plane strain modeling fixture with transparent wall. Note the chip ejection in front of the slider for the wax simulation.

to the applied load for single pass experiments. At very low loads (Regime I) there is plastic deformation but no visible wear debris. At intermediate loads and intermediate penetration (Regime II) there is plastic deformation around the diamond slider, from the center of the track toward the outside-side flow. A built up ridge is observed at the outside edges of the wear track and occasionally a wear particle is seen to separate from the bulk. At higher loads (Regime III) a semicontinuous chip is evolved in front of the diamond. This chip appears to be made up of discrete platelets which have adhered to one another. In addition to the front flow in Regime III there is substantial side flow present. A pictorial definition of each wear regime is given in Fig. 3.

The modeling experiments show clearly the definitions of Regimes I and III and give insight into the processes of Regime II. Figure 4 shows the equivalent of Regime I with the brass cylinder sliding on modeling clay. At light penetration and consequently light loads, the clay "recovers" plastically behind the slider. This can be scen in the grid patterns and by measuring the recovered height of the clay. Figure 5 shows the equivalent of Regime III in modeling clay. It is apparent that the plastic constraint induced by the slider forces the removal of material ahead of the slider. This is analogous to large negative rake machining without a relief face on the tool and analogous to impact erosion ${ }^{11}$.

The front flow chipping mechanism prevalent in the clay is shown in sequential order in Fig. 6. Video taped replays show that the process starts when 


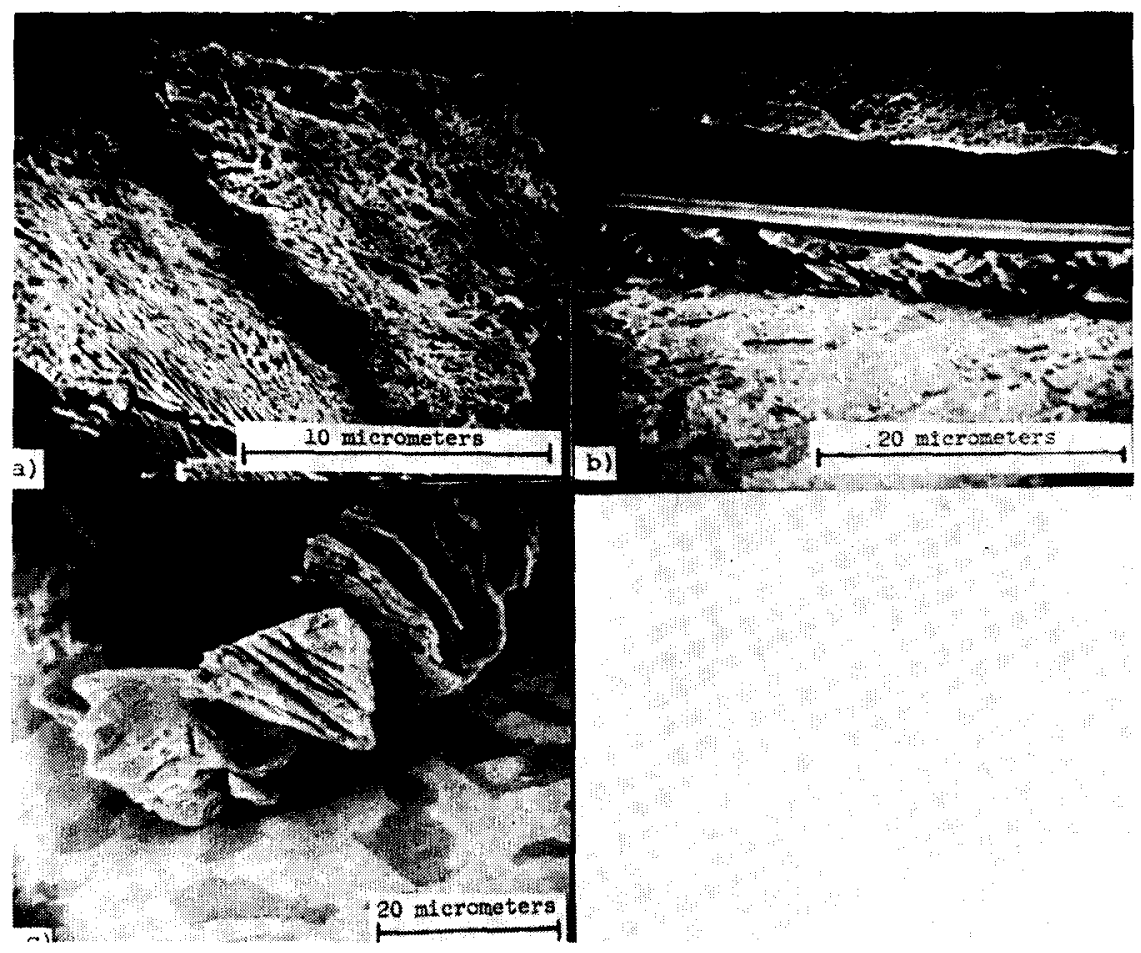

Fig. 3. (a) Regime I. The etch marks are blurred over in the wear track (b) Regime II. Wear debris builds up along the sides of the track. (c) Regime III. Wear chips are ejected ahead of the slider.

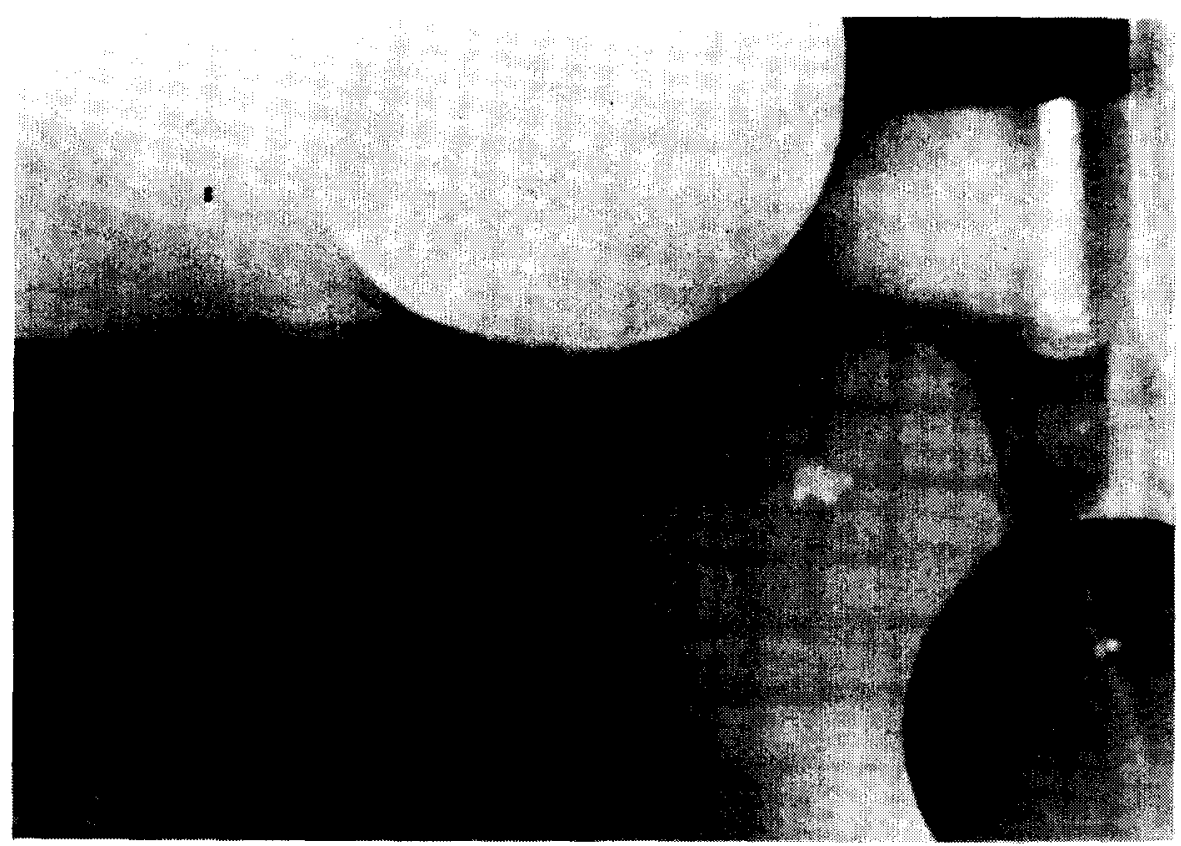

Fig. 4. Plastic recovery behind the slider at light penetration. Shown in gridded modeling clay. 


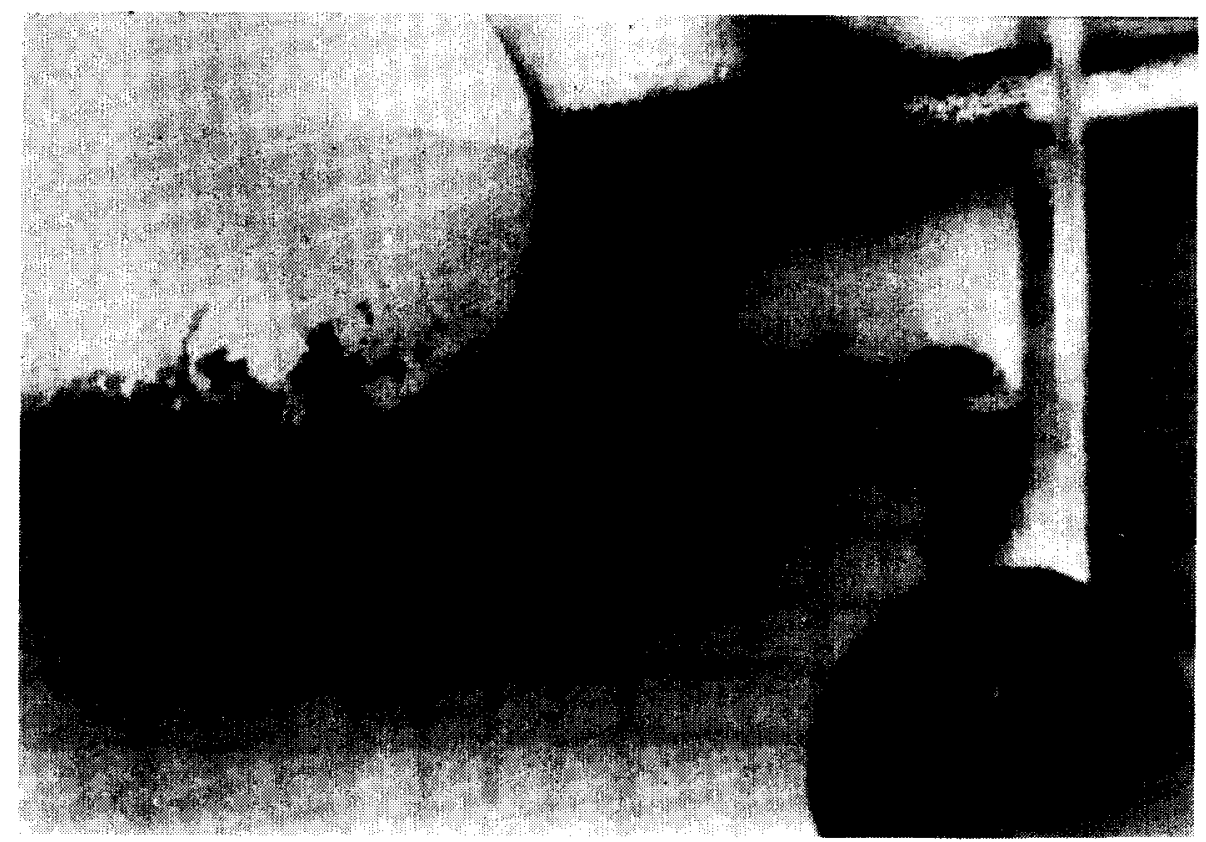

Fig. 5. Wear chips evolved ahead of the slider in Regime III simulation.

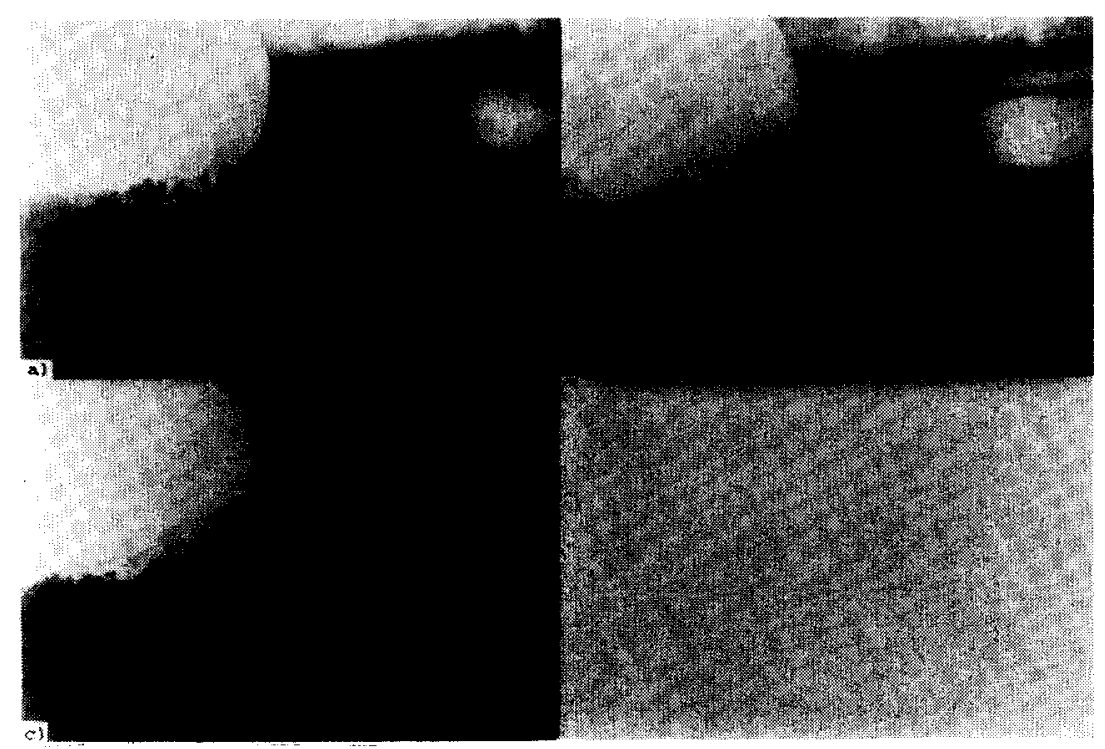

Fig. 6. Regime III flow mechanics in modeling clay. (a) Bulge formation. (b) Crack propagation. (c) Chip ejection.

the slider travels a critical distance building up a bulge ahead of the slider (Fig. 6(a)). Johnson and Rowe ${ }^{12}$ have demonstrated a similar effect previously in light reduction extrusion of metals through low angle dies. When the bulge in the 
clay reaches a critical height a shear crack starts to form at the junction of the original surface and the bulged surface (Fig. 6(b)) and propagates into the substrate along a plane roughly parallel to the surface of the slider. As the crack proceeds the platelet or chip is forced up along the surface of the slider where it adheres, under high compressive stress, to the next platelet (Fig. 6(c)). This behavior was shown to occur in modeling experiments done with cast paraffin wax (Fig. 7) as well as the in situ pin-on-disk experiments (Figs. 8 and 9). Because of the constraints to side flow induced by the modeling fixture Regime II (side flow regime) was impossible to model with clay or wax. Review of the video tapes of the pin-on-disk experiments indicates, however, that the same bulge formation mechanism applies in Regime II with an altered flow geometry.

The separation of wear behavior into Regimes I, II, and III is easily explained in terms of the flow behavior of the softer material relative to the constraint offered by the harder. At very low loads and consequently low penetrations plastic recovery behind the slider is possible. Johnson and Sowerby ${ }^{13}$ explain that for similarly light

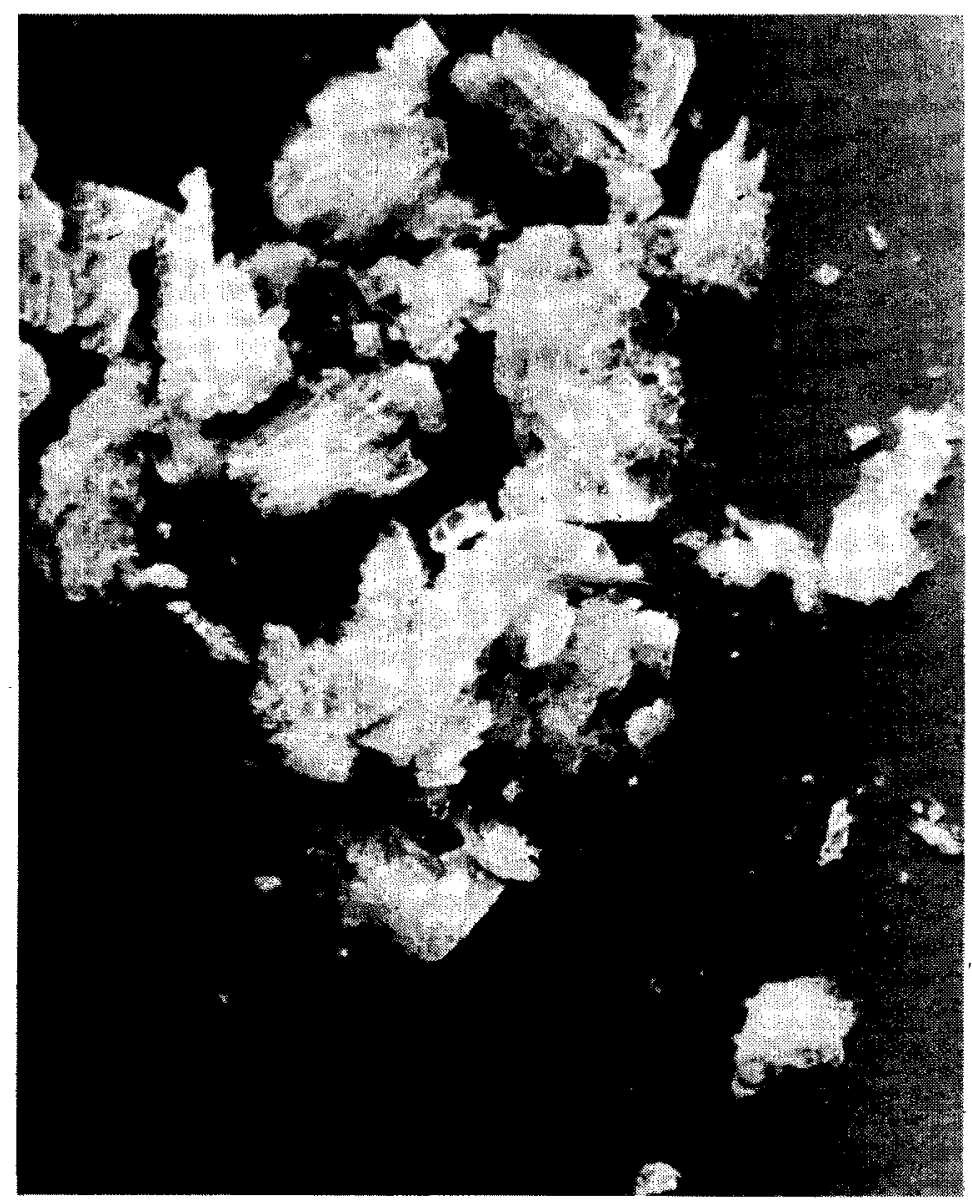

Fig. 7. Platelike debris collected from modeling fixture during Regime III wax simulation. 


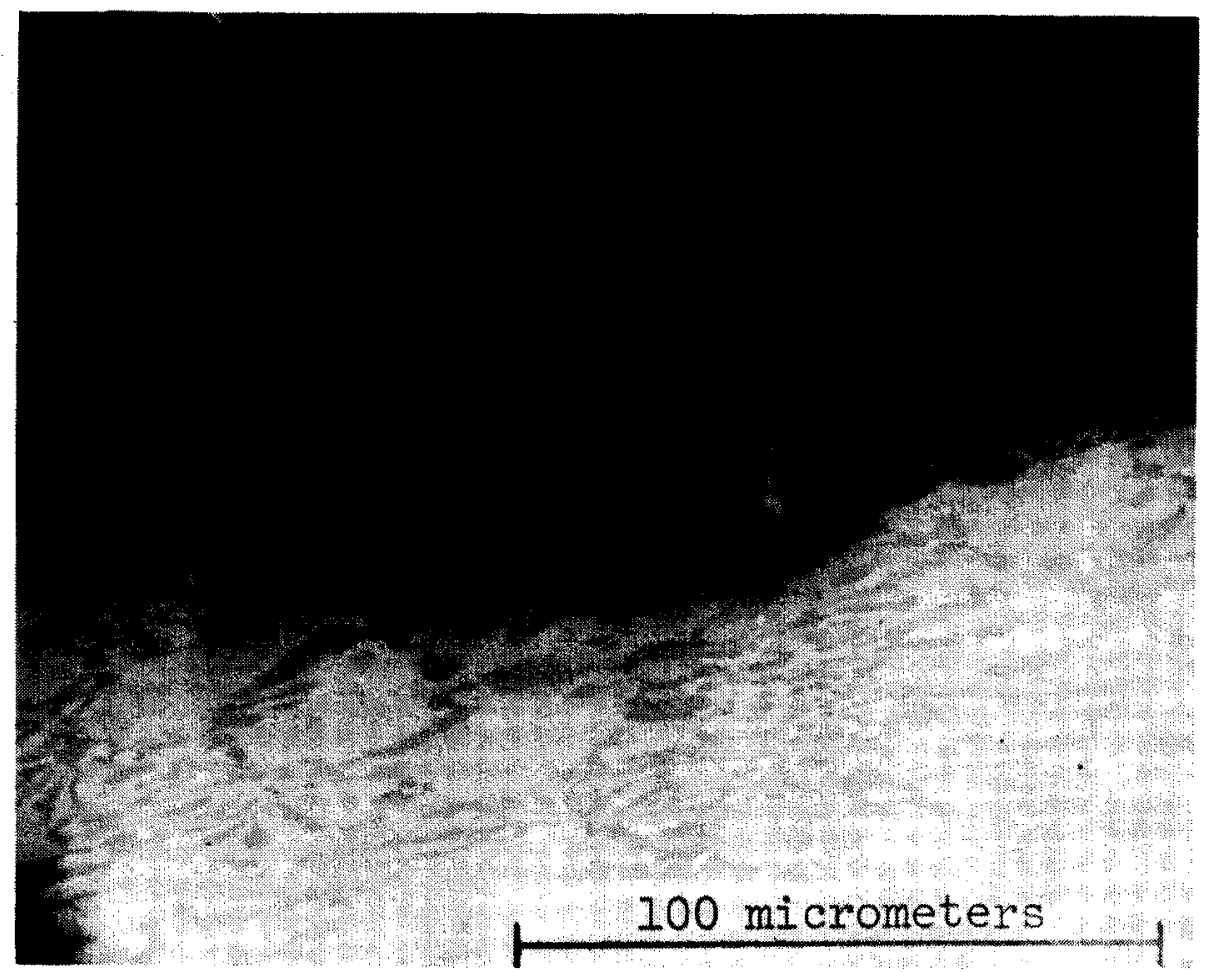

Fig. 8. Wear of diamond on white cast iron. Note the platelike form of the debris and the ejection ahead of the diamond slider.

reductions during plate extrusion plastic recovery must take place behind the die. It is clear that the similar geometric constraints lead to the behavior shown in Regime I. At intermediate penetrations, however, material is forced around the sides of the slider in a flow pattern requiring less work than complete recovery behind the slider. Finally, at large penetrations plane strain conditions are met at the center of the contact track due to pressure exerted normal to the track by the surrounding material. This constraint combined with the continuity condition required behind the slider causes ejection of wear debris ahead of the slider.

The results of this research give an alternative explanation for the findings of $\operatorname{Suh}^{6}$ and his associates ${ }^{7}$. It is quite apparent that the platelike wear debris found in this study is the result of plastic flow combined with surface initiated shear cracks required by the constraint induced by the slider. No void coalescence was observed in modeling clay and wax. Yet wax and clay appear to form debris in the same manner as steel and white iron for similar constraints. Clearly, in these experiments a plastic flow mechanism and not delamination causes platelike debris to form. It must also be seen from the sequence of events leading to the formation of a wear particle (Fig. 6) that the shear fracture properties must be included in any quantitative model of penetrative wear.

Slip line field analysis

Repeated observation of the flow and fracture process indicated in Fig. 6 


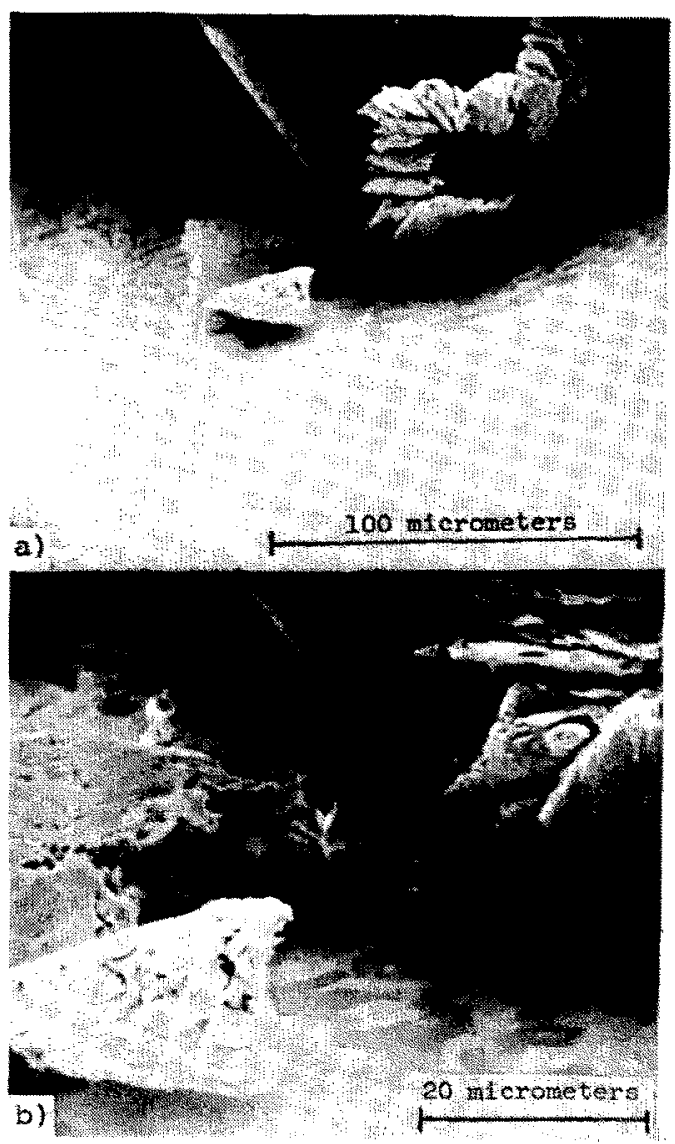

Fig. 9. Wear of diamond on white cast iron. (a) Wear plates adhere to one another and form a semi-continuous chip. (b) Close up of (a) showing the smooth top surface of the debris coming off the face of the diamond.

leads to the approximate slip line analysis shown in Fig. 10. Figure 10(a) shows the approximate field during bulge formation. While the angles $\phi, \psi$, and $\alpha$ can be determined experimentally for clay it is clear that they are functions of the relationship of the flow stress to the shear fracture stress. Hence, while modeling clay is traditionally used to show flow patterns during metal plasticity the angles mentioned above will not necessarily be similar for different materials. Thus the shape of the debris in other materials could be different while the mechanics remain essentially the same. In fact, the particle shape for wax, steel and white cast iron was roughly platelike (as opposed to triangular for clay) but the debris formation process is apparently the same. Figure 10(b) shows the severe shear taking place along the shear fracture line. This is shown vividly in modeling clay in Fig. 6(b). Figure 10(c) shows the last stage of the wear mechanism, the ejection of the particle and the subsequent reindentation of the new surface. Again the angles $\phi$ and $\psi$ are material specific; $\alpha$ however, is equal to 45 degrees.

While the slip line fields shown in Fig. 10 do not have compatible hodograph 

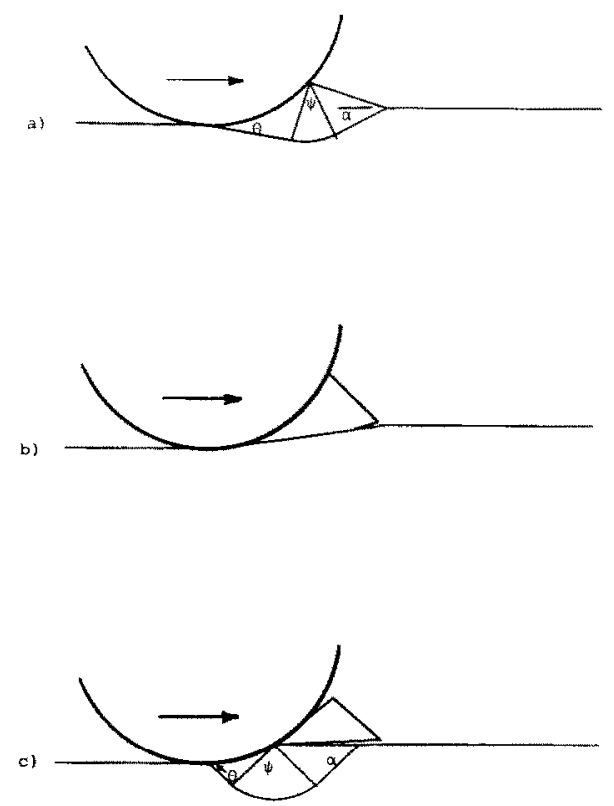

Fig. 10. Approximate slip-line fields for Regime III wear particle formation, (a) A bulge forms ahead of the slider. (b) $\Lambda$ crack propagates from the surface into the substrate. (c) The chip is ejected ahead of the slider. These fields correspond to the flow patterns seen in Fig. 6.

solutions, this is to be expected since they do not represent steady state flow. The fields in Fig. 10 do represent incipient flow tendencies. It should be noted that all three fields show a shear boundary condition on the trailing edge of the slider as shown in the modeling studies.

\section{CONCLUSIONS}

A mechanism for the formation of plate-like wear debris found in penetrative wear has been proposed. These particles form in a three part process. First, as sliding proceeds a bulge forms ahead of the slider. Second, a shear crack initiates at the surface and propagates into the substrate parallel to the slider face. Third, the platelet is pushed up the face of the slider and attaches to next particle. In situ scanning electron microscope experiments and work with modeling clay and wax confirm this analysis. A series of slip line fields has been presented to approximate the flow patterns of the wear process. The shear crack that occurs indicates the need for future wear models to include fracture toughness in shear for quantitative or comparative analysis.

\section{REFERENCES}

1 D. Tabor, Junction growth in metallic friction: the role of combined stresses and surface contamination, Proc. Roy. Soc. (London), 251 A (1959) 378. 
2 J. F. Archard, Wear, Interdisciplinary Approach to Friction and Wear, NASA SP-181, Washington, D.C., 1968.

3 M. Antler, Processes of metal transfer and wear, Wear, 7 (1964) 181.

$4 \mathrm{M}$. Cocks, The formation of wedges of displaced metal between sliding metal surfaces, Wear, 8 (1965) 85.

5 M. Cocks, Shearing of junctions between metal surfaces, Wear, 9 (1966) 329.

6 N. P. Suh, The delamination theory of wear, Wear, 25 (1973) 111 ,

7 S. Jahanmir, N. P. Suh and E. P. Abrahamson, Microscopic observations of the wear sheet formation by delamination, Wear, 28 (1974) 235.

8 R. C. Bill and D. W. Wisander, Role of plastic deformation in wear of copper and copper $-10 \%$ aluminum alloy in cryogenic fuels, NASA TN D-7253, April (1973).

9 W. A. Brainard and D. H. Buckley, Dynamic friction and wear studies in the scanning electron microscope, NASA TN (1974) (in preparation).

$10 \mathrm{R}$. B. Gundlach, Microstructure, hardness and abrasion resistance of as-cast $17.5 \mathrm{Cr}$ White Irons, Proc. Amer. Foundrymen's Soc. Cast. Congr., May, 1975.

$11 \mathrm{~J}$. M. Hutchins and R. E. Winter, Particle erosion of ductile metals: a mechanism of material removal, Wear, 27 (1974) 121.

$12 \mathrm{R}$. W. Johnson and G. W. Rowe, Bulge formation in strip drawing with light reductions in area, Proc. Instn. Mech. Engrs., $182(1)(22)(1967-68) 521$.

13 W. Johnson, R. Sowerby and J. B. Haddow, Plane-Strain Slip-Line Fields, Elsevier, New York, 1970. 